\section{The 3Rs: Reduction and refine- ment through a multivariate statistical analysis approach in a behavioural study to unveil anxiolytic effects of natural extracts of Tilia tomentosa}

Guendalina Olivero, ${ }^{1}$ Federica Turrini, ${ }^{1}$ Matteo Vergassola, ${ }^{1}$ Raffaella Boggia, ${ }^{1}$ Paola Zunin, ${ }^{1}$ Dario Donno, ${ }^{2}$

Gabriele Loris Beccaro, ${ }^{2}$ Massimo Grilli,1,3 Anna Pittaluga1,3

1Department of Pharmacy (DIFAR), University of Genoa, Genoa; 2Department of Agriculture, Forestry and Food Science, University of Torino, Turin; 3Inter-University Center for the Promotion of the 3Rs Principles in Teaching and Research, Italy

\begin{abstract}
We propose a multivariate statistical approach based on Principal Component Analysis (PCA) as an useful instrument to improve the Rules of Refinement and Reduction in in vivo animal experimentation. We analysed with PCA the preliminary data from a study on the effects of the oral administration of Tilia tomentosa bud extracts (TTBEs) on the behavioural skills of adult and aged male and female mice. PCA allows to rationalize the data set information and to dissect the results, showing connections among variables under study (behavioural parameters) and different trends in the experimental groups (control and TTBEs-administered animals). Our results show that PCA can give some important information that can be useful for the refinement of the experimental protocol, in order to reduce the number of the animals used in the experiments and/or the behavioural tests to get reliable information.
\end{abstract}

\section{Introduction}

The principles of the 3Rs (Reduction, Refinement and Replacement) are at the basis of an ethical use of animals in scientific research. Since many years, we have made efforts to set up our experimental protocols according to the ARRIVE guidelines and the $3 R s$, adopting strategies such as minimizing the number of animals to get strong results and sharing tissues between research groups.
We here propose a multivariate statistical approach based on Principal Component Analysis (PCA) applied as a useful instrument to improve the rules of refinement and the reduction in in vivo animal experimentation.

Starting from 2018, our research group was involved in a project called FINNOVER ( $\left.n^{\circ} 1198\right)$, within the Interreg ALCOTRA Italy/France trans frontier call, dedicated to the study of the effects of an in vivo oral administration of bud derivatives in aging. The study was so far focused on investigating the impact of Tilia tomentosa bud extracts (TTBEs) on adult (3-6 months old) and aged (20-22 months old) male and female mice.

In 2015, Allio et al. investigated whether TTBEs affect hippocampal Gamma-aminobutyric acid (GABA)ergic synapses. ${ }^{1}$ Their results demonstrated that a direct application of TTBEs on post-synaptic terminals can activate a chloride-mediated current that is blocked by bicucullin, picrotoxin and flumazenil. These data suggest that TTBEs can amplify the $\mathrm{GABA}_{\mathrm{A}}$ mediated signaling, mimicking GABA and benzodiazepines.

Based on the efficacy of TTBEs on $\mathrm{GABA}_{\mathrm{A}}$ receptors, we investigated the effects of the oral administration of TTBEs on the mice behavioural skills related to anxiety, curiosity and spontaneous motor activity in the hole-board and in the lightdark box tests, two experimental protocols useful for quantifying these behavioural parameters in animals.

In an attempt to underline changes in the behavioural responses due to the TTBEs administration, the data obtained from a preliminary set of experiments were first analyzed by using PCA. PCA, the most commonly used chemometric technique, is an unsupervised pattern recognition technique useful to rationalize the data set information. It allows to dissect the results, showing the connections among the variables under study (behavioural parameters) and the potential different trends in the experimental groups. We predicted that PCA can be useful for the refinement of the experimental protocol to rationalize the information of the data set obtained from each animal group and possibly to significantly reduce the number of the animals and/or of the behavioural tests to obtain reliable information.

\section{Materials and Methods}

Adult (3-6 months) and old (20-22 months) male and female mice were randomly assigned to three different groups:
Correspondence: Anna Pittaluga, Department of Pharmacy (DIFAR), University of Genoa, Genoa, Italy.

E-mail: pittalug@difar.unige.it

Key words: Mice; refining; reducing.

Conference presentation: this paper was presented at the Second Centro 3R Annual Meeting - 3Rs in Italian Universities, 2019, June 20-21, University of Genoa, Italy.

Received for publication: 28 October 2019. Accepted for publication: 11 November 2019.

This work is licensed under a Creative Commons Attribution NonCommercial 4.0 License (CC BY-NC 4.0).

\section{(C) Copyright: the Author(s), 2019}

Licensee PAGEPress, Italy

Biomedical Science and Engineering 2019; 3(s3):116 doi:10.4081/bse.2019.116

water-administered mice (control), vehicleadministered mice (EtOH /glycerin /H2O), TTBEs-administered mice $(\mathrm{n}=6$ animals for each experimental group). In order to reduce stress in mice, TTBEs $(1 \div 2000$ dilution) were dissolved in the drinking water. Animals were under treatment for 14 days and monitored for the daily drinking volume intake and for the gain of weight. The behavioural tests were performed before and at the end of the treatment.

Data from the behavioural analysis were analyzed using PCA. PCA was performed by NIPALS algorithm ${ }^{2}$ on male and female data matrix (6 rows and 5 columns) respectively, to quickly screen and rationalize the information of the experimental data. The variables under study were: $n^{\circ}$ of head dippings, $\%$ of explored area and $\%$ of entries into the centre (hole-board test); $n^{\circ}$ of transitions and time in light (light-dark box test). Autoscaling pre-treatment (column centering + column scaling) was performed in order to normalize the data, adapting the different measure units of variables. An R-based chemometric software developed by the Group of Chemometrics of the Italian Chemical Society (freely downloadable from gruppochemiometria.it /index.php/software, 2018) was used to perform the multivariate data analysis.

\section{Results}

The univariate analysis of the preliminary results from the first set of experiments showed that TTBEs affects curiosity, anxiety and the spontaneous motor activity in 
mice, depending on their age and gender. Differently, PCA of these preliminary results led to some important information helpful for the refinement of the experimental protocol.

In male mice, the PCA unveiled that young male mice diverged from the old ones on PC1 (which explains almost the $60 \%$ of the whole data set information) and that TTBEs treatment made old mice more similar to the young ones. Furthermore, both in old and in young male mice, the animals treated with TTBEs were separated from the others, while the water and vehicle treatments carried the same information on PC1 and PC2 (which together explain almost the $87 \%$ of the total information). This would indirectly suggest the possibility to eliminate one experimental group (the control or the vehicle-administered ones), so reducing the numbers of mice used in the experiments. Last but not least, on PC1 the time in the light and \% of entries into the centre carried out the same information as the curiosity and the anxiety are concerned, compatible with the conclusion that just one behavioral test could be enough for highlighting the TTBEs effect.

In female mice, PC1 (which explains the $47 \%$ of the total information of the data set) separated young mice from the old ones. The latest group was characterized by a smaller number of entries into the centre when compared to young mice. Furthermore, in the group of young female mice, the ones treated with TTBEs were separated from the others, mainly on PC2 (which explains the $40 \%$ of the total information). Actually, TTBEs treatment increased the number of transitions in young female mice. However, differently from what highlighted in male mice, the vehicle influenced the behaviour in old female mice similarly to TTBEs, but differently from water.

\section{Conclusions}

The PCA analysis permits an overall view of the results from an experimental paradigm, highlighting the relations between objects (the animals) and variables (behavioural parameters) under study. This approach can be used to improve the refinement of the applied experimental protocol, by excluding variables that give the same information and so reducing the number of animals to be used within the tests.

\section{References}

1. Allio A, Calorio C, Franchino C, et al. Bud extracts from Tilia tomentosa Moench inhibit hippocampal neuronal firing through GABAA and benzodiazepine receptors activation. J Ethnopharmacol 2015;172:288-96.

2. Wold S, Esbensen K, Geladi P. Principal component analysis. Chemometr Intell Lab Syst 1987;2:37-52. 